\title{
Thromboprophylaxis with low dose unfractionated heparin plus compression stockings reduces thromboembolic complications of colorectal surgery
}

\author{
Wille-Jørgensen P, Rasmussen MS, Andersen BR, et al. Heparins and mechanical methods for thromboprophylaxis in \\ colorectal surgery. Cochrane Database Syst Rev 2001;(4):CD001217 (latest version May 26 2001).
}

\section{QUESTION: In patients having colorectal surgery, what is the effectiveness of prophylactic methods using heparin and mechanical methods on reducing postoperative thromboembolism (TE)?}

Source of funding: $i$ Hospital Corporation

For correspondence: Dr P Wille-Jørgensen, Department of Surgical Gastroenterology K, Bispebjerg Hospital, Copenhagen NV,

Denmark.

peerwille@vip.cybercity.dk

\section{Data sources}

Studies were identified by searching Medline, EMBASE/ Excerpta Medica, and the Cochrane Library (1970 to May 2000). Meeting abstracts and reference lists of reviews were handsearched.

Prophylactic methods for reducing deep venous thrombosis or pulmonary embolism or both at 7-14 days after colorectal surgery*

\begin{tabular}{lllll} 
Comparisons & $\begin{array}{l}\text { Weighted } \\
\text { event rates }\end{array}$ & RRR (95\% Cl) & NNT (Cl) \\
\hline Any heparin $v$ no treatment or placebo & $5.5 \%$ v $16.7 \%$ & $60 \%(38$ to 74$)$ & 9 (7 to 16) \\
\hline & & $\operatorname{RRI}(\mathrm{Cl})$ & NNH (Cl) \\
\hline LDH alone $v$ LDH and compression stockings & $21.1 \%$ v 5.1\% & $314 \%(22$ to 1304) & 7 (4 to 28) \\
\hline
\end{tabular}

${ }^{*} \mathrm{LDH}=$ low dose unfractionated heparin. Other abbreviations defined in glossary; RRR, RRI, NNT, NNH, and $\mathrm{Cl}$ calculated from data in article.

\section{COMMENTARY}

Colorectal surgery appears to be associated with a higher risk of thromboembolism than general surgery. ${ }^{12}$ This may be related to preoperative factors associated with a greater risk, such as cancer and inflammatory bowel disease; however, there are wide variations in thromboprophylactic regimens used. ${ }^{3}$

The findings of Wille-Jørgensen et al reinforce those of reviews in other fields; heparin reduces the odds of thromboembolism by at least half, and the addition of graduated compression hosiery is more effective than heparin alone. No difference exists between unfractionated and LMWH for thromboembolic events, but WilleJørgensen et al were unable to extract data on bleeding complications. Other reviews have found that LMWH results in greater reductions in the risk of minor bleeding complications than unfractionated heparin. ${ }^{4}$

2 major methodological weaknesses of this review deserve mention. Firstly, because few trials enrolled colorectal patients alone, Wille-Jørgensen $e t$ al extracted data on colorectal subgroups from studies of general surgical patients to increase statistical power, potentially introducing selection bias. Secondly, 66 colorectal and general surgery studies were identified for inclusion, but data could be obtained from fewer than a third of these (ie, authors of original studies failed to respond to the reviewers' requests for information). Despite these problems, the findings are consistent with those of other reviews and are biologically plausible; thus, current evidence justifies the use of both heparin and graduated compression hosiery for preventing thromboembolism in patients having colorectal surgery.

Andrew Jull, RN, MA Clinical Nurse Consultant Auckland Hospital Auckland, New Zealand

1 Wille-Jørgensen P, Ott P. Predicting failure of low-dose prophylactic heparin in general surgical procedures. Surg Gynecol Obstet 1990;171:126-30.

2 Huber O, Bounameaux H, Borst F, et al. Postoperative pulmonary embolism after hospital discharge. An underestimated risk. Arch Surg 1992;127:310-3.

3 Caprini JA, Arcelus JI, Hoffman K, et al. Prevention of venous thromboembolism in North America: results of a survey among general surgeons. J Vasc Surg 1994;20:751-8.

4 Palmer AJ, Schramm W, Kirchhof B, et al. Low molecular weight heparin and unfractionated heparin for prevention of thrombo-embolism in general surgery: a meta-analysis of randomised clinical trials. Haemostasis 1997;27:65-74.

\section{Study selection}

Studies were selected if they were randomised controlled trials (RCTs) or controlled clinical trials; compared prophylactic interventions using heparins or mechanical methods or both with each other or placebo; included patients $>18$ years of age having major colorectal surgery; diagnosed TE objectively using ascending venography, the 125-I-fibrinogen uptake test, ultrasound or Doppler methods, pulmonary scintigraphy, or autopsy; and reported start and end times of prophylaxis use. Studies were excluded if the only diagnostic measure used was a thermographic method, isotopic method other than the 125-I-fibrinogen uptake test, plethysmographic method, or purely clinical method.

\section{Data extraction}

Data were extracted on patient characteristics, methodological quality, prophylaxis type, and outcomes including deep venous thrombosis (DVT), pulmonary embolism (PE), TE, and mortality. Exclusive data on colorectal patients and 30 day mortality were sought from authors if not reported.

\section{Main results}

19 studies met the selection criteria. 3 studies reported data on colorectal patients only, and 16 studies had extractable data for colorectal patients. 10 studies (641 patients) compared some type of heparin with no treatment or placebo; 4 studies (1183 patients) compared low dose unfractionated heparin (LDH) with low molecular weight heparin (LMWH); and 4 studies (130 patients) compared various combinations of mechanical methods. The screening methods used for DVT detection were the 125-I-fibrinogen uptake test (15 studies), mandatory venography (1 study), and Doppler ultrasound (3 studies). DVT and PE were analysed at postoperative times of about 7-14 days using a fixed effects model. 11 studies (641 patients) showed that any heparin was more effective than no treatment or placebo in preventing DVT, PE, or both (table). 4 studies (1183 patients) showed that LDH and LMWH did not differ for DVT and PE prevention (8.6\% v 8.5\%, respectively). 2 studies (111 patients) showed that $\mathrm{LDH}$ alone was associated with a higher risk for DVT or PE or both than LDH plus compression stockings (table).

\section{Conclusions}

In patients having colorectal surgery, low dose unfractionated heparin $(\mathrm{LDH})$ plus compression stockings is a more effective thromboprophylactic than LDH alone. $\mathrm{LDH}$ and low molecular weight heparin do not differ for thromboprophylactic outcomes. 